\title{
Multiple Sclerosis: Assessment of Disease Progression and Effects of Treatment
}

\author{
D.W. Paty
}

\begin{abstract}
MS could well be a two stage disease. The first stage involves the sequential development of multiple small lesions, mostly inflammatory, that accumulate at a given rate. The second stage could be that of consolidation and confluence of lesions that involves not only demyelination but gliosis. MRI now gives us an opportunity to watch the evolution of these processes and also to monitor treatment effects. It is only after the evolution of this process is understood that we can design rational therapies directed toward the prevention of spasticity in MS.
\end{abstract}

RÉSUMÉ: La sclérose en plaques: surveillance de la maladie progressive et les résultats du traitement La sclérose en plaques pourait bien être une maladie en deux stages. Le premier stage comporterait le développement séquentiel de multiples petites lésions, de nature inflammatoire pour la plupart, se développant à un rythme donné. Le deuxième stage pourrait être la consolidation et la confluence des lésions impliquant non seulement la démyélinisation mais aussi la gliose. L'IRM nous fournit maintenant l'occasion de surveiller l'évolution de ces processus et également les effets du traitement. Ce n'est que lorsque l'on comprendra l'évolution de ce processus que nous pourrons élaborer des thérapies rationnelles dirigées vers la prévention de la spasticité dans la SEP.

Can. J. Neurol. Sci. 1987; 14:518-520

Multiple sclerosis is a disease of young adults in which a genetically susceptible individual apparently develops an autoimmune process that causes demyelination. The genetic susceptibility appears to be most frequent in persons of northern European racial origin, and least in those of African racial origin. The clinical course is marked by relapses and remissions of neurological deficits which come and go in an unpredictable fashion. The clinical relapses seem to be very dependent upon the anatomical location of the current disease activity. Since there are many areas of the nervous system, particularly the cerebral hemispheres, that are "silent" in terms of clinical expression, one might think that in a disease such as MS there might well be a great deal of disease activity that is not reflected in clinical symptoms.

Pathologists have observed for years that the extent of disease as detected at autopsy doesn't seem to have much relationship to the clinical description of disease activity in that same patient. The first event in the production of the MS lesion may well be a breakdown in the blood brain barrier (BBB). CT scanning has shown that, without a doubt, there are focal and widespread areas of disruption in the BBB. 'Many of these areas of $\mathrm{BBB}$ disruption are asymptomatic. It is also known that these abnormal areas can come and go without leaving any detectable abnormality on the CT scan. Following breakdown in the BBB there is apparently a great deal of inflammation, and this inflammation tends to be distributed in a perivenous fashion. ${ }^{2,3}$ Anatomically the most active areas appear to be surrounding the lateral ventricles. It is not known whether the inflammatory process is directed against the myelin or the oligodendrocyte. In any event, the eventual result of the inflammation appears to be demyelination. The local area of demyelination appears to be characterized by sharp borders. The number of oligodendrocytes is decreased in areas of demyelination, but some investigators have found that the number of oligodendrocytes in areas surrounding the demyelination may be actually increased. ${ }^{2}$

Following the development of demyelination, the nervous system reacts by the production of astrocytic gliosis. Just exactly what the trigger for the development of gliosis may be is not known, but there must be mitogenic factors which stimulate astrocytic proliferation. In addition there may be some elements of remyelination as well. Remyelination in the central nervous system (CNS) was considered to be impossible only a few years ago. Recent experiments ${ }^{4}$ have now shown that remyelination can occur in the CNS if the process that initiated demyelination is halted before the actual death of the oligodendrocyte. Just exactly how much remyelination can contribute to MS is not known, but pathologists have now begun to detect evidence of remyelination more and more frequently in the examination of MS brains. ${ }^{5}$

Magnetic resonance imaging (MRI) has now begun to show us a great deal about the evolution of the disease process in MS. 
Serial MRI studies have now shown that areas of abnormality can develop very quickly, and then begin to fade over a period of a few weeks to months. ${ }^{6}$ Most of these new areas of abnormality are asymptomatic. Out of 17 patients that have been studied by our group to date, there were 17 new areas of abnormality that occurred in 10 patients, each of whom had been studied every two weeks in a serial fashion over six months time. There were many more MRI events than there were clinical ones, and in none of the clinical events was an appropriate MRI lesion seen.

Just exactly what the pathological process may be that is producing these new lesions is not yet known. The fact that the abnormal areas begin to reduce in size over a period of only a few weeks suggests that this process is not involving demyelination and subsequent remyelination, though we cannot be absolutely sure.

Attempts at qualitative MRI analysis in MS have now shown that the extent of the process can be measured. The concept of quantitative measurement of the extent of MS as detected by MRI is now being used as a way of adjudicating clinical trials. ${ }^{8}$ One would assume that if a new treatment is effective, that it would slow down or stop the accumulation of new lesions or reduce the tendency for pre-existing lesions to enlarge. A computer assisted method of area measurement, slice by slice, has been developed and in serial studies this has shown that in untreated patients the extent of abnormality increases over time.

Using this particular method to examine the differences between benign and chronic progressive disabled patients, matched by age, sex and duration of disease $e^{9}$ has shown that one of the major differences between benign disease and chronic progressive disabling disease is the presence of confluence of lesions around the ventricles. There is also a tendency for benign patients to have many more asymptomatic lesions than do chronic progressive patients. Does this mean there is a second process, independent of demyelination, that influences whether or not there will be chronic disability in MS? One candidate for such a process might be the development of severe degrees of astrocytic gliosis. Fog ${ }^{10}$ in 1969 put forward the idea that gliosis might well contribute to the chronic progressive deficit in disabling MS. This idea would then suggest that relapsing and remitting MS is a disease of inflammation and demyelination and that in chronic progressive MS the same elements are present, but a second element, that of gliosis, is then introduced which goes on to contribute in a major fashion to the development of chronic disability and spasticity.

Pathological correlation studies examining the similarities and differences between the MR image and the actual brain pathology have now shown that MRI might well be able to detect differences in degree of gliosis. " If this is indeed so, MRI could be used as a method for following the disease process, detecting the time when gliosis might appear, and suggesting when the appropriate time might be to use new disease strategies directed toward inhibiting astrocytic proliferation.

Therefore a strategy for the prevention of spasticity in MS might involve vigorous immunosuppressive therapy on the one hand, and a new concept directed toward the inhibition of gliosis on the other.

\section{How do We Assess The Effect Of A New Therapy?}

The standard way to evaluate therapy in a neurological disease is to establish an ordinal scale that will reflect the degree of neurologic impairment or functional disability. Impairment is that aspect of neurological dysfunction that is detected by the neurological examination. In a diagnostic sense, impairment reflects the areas of the nervous system that are abnormal. The degree of impairment would therefore seem to reflect the intensity of the disease process at any one particular point. Disability is a measurement of the functional incapacity that patients have as a result of their impairment. This kind of abnormality causes problems in the activities of daily living, and it is, of course, that area which is of most concern to the patient.

Unfortunately many neurological evaluation schemes confuse the measurement of impairment with the measurement of disability. A plea should be made to separate the two types of measurement so that in a semi-quantitative sense the clinician can measure either neurological abnormalities (impairment) or measure the functional consequences of that impairment (disability).

As mentioned above, the usual way that neurologists evaluate patients is to assign a scale in which a minor level of abnormality is graded as 1 , and more severe degrees of abnormality are graded with higher numbers. A scale that is organized in this fashion guarantees that assignment of the number 2 means that the deficit is greater than that of number 1 . There is no guarantee that there is an equal distance between 1 and 2, and 2 and 3 . This means that the ordinal scale is very different from a linear one. The linear scale guarantees that the degree of change of one digit on the scale is the same between 1 and 2,2 and 3, etc. This means that such a measurement can be used in statistics to determine means, plot curves, and do correlation coefficients. Unfortunately an ordinal scale does not allow that kind of statistical manipulation.

The advent of MRI into the MS arena may change our ability to do objective and linear measurements of the extent of disease. MRI can reveal for the first time the extent of the MS process in the nervous system. Initially the ability to measure extent of disease by MRI was confined to the cerebral hemispheres, but new machines can now image the spinal cord. This means that the entire CNS can now be surveyed for MS abnormalities. Strategies for detecting the extent of the process by tracing the outline of lesions, and adding the abnormalities slice by slice are now being used in the adjudication of clinical trials. ${ }^{8}$

The hypotheses are as follows: The natural progression of the disease process in MIS must involve 1) new areas of abnormality, 2) enlargement and increasing intensity in previously existing areas of abnormality, and 3) a combination of 1) and 2). MRI can now detect the extent of the process with an accuracy that should lend itself to the measurement of both the rate of formation of new lesions and the degree of change in extent of lesions. These will be discussed independently as follows:

\section{Rate of Formation of New Lesions}

The relapsing and remitting clinical course of MS has suggested for years that not only are new areas of the nervous system being involved over time, but there is some sort of resolution process going on. Serial MRI studies ${ }^{6.7}$ have now shown that the rate of accumulation of new lesions is considera- 
bly greater than that of the clinical relapse rate. More extensive investigation is needed, but perhaps one method of assessing the rate of formation of new lesions might be to do serial prospective MRI scans. Our studies in this area have suggested that when new lesions form they can appear and become maximum within a period of two weeks to a month. They then tend to gradually resolve over the next four to eight weeks. This finding would suggest that a rate of one scan each month, could be expected to pick up the majority of new lesions. This could now be used as a method of measuring therapeutic effect. The assumption is that these new lesions are basic to the underlying MS process, and that if one could stop or slow down the rate of accumulation of these lesions one could affect the later development of clinical disability. Since most of these new lesions are asymptomatic, a simple strategy of monthly MRI scans, with careful repositioning to make sure that one is not missing lesions or seeing additional lesions because of repositioning artifact, and simply counting the rate of formation of new lesions could be used. This would give an objective measure, apparently much more sensitive than the clinical examination, of detecting differences between treatment groups.

\section{Quantitative Assessment of the Extent of Disease}

The ability to accurately measure the extent of the process is also aided by MRI. The method of tracing out the area of abnormalities has obvious limitations, but serial studies over time should give an accurate reflection of major changes in the extent of disease. If putative therapies are to be adjudicated over a relatively long period of time, entry and exit MRI studies should give a fairly clear index of disease progression. Newer MRI technologies, especially with thin slices, will reduce the error due to repositioning and partial volume effect. This would then presumably give a more accurate assessment of therapeutic effect than would simple neurological impairment rating.

This does not mean that the neurological rating scales must be abandoned. It is only to provide additional evidence for therapeutic effectiveness, and perhaps give clues that might not be detectable by clinical methods alone.

\section{TheraPIES CURRENTLY USED IN MS}

Current attempts at MS therapy are usually directed toward arresting the pathological process. ${ }^{12}$ Most of these therapeutic modalities have been directed toward an immunological pathogenesis. These include anti-inflammatory agents such as adrenocorticotropic hormone (ACTH) and corticosteroids. Non steroidal anti-inflammatory agents have not generally been used or found effective.

Immunomodulatory agents such as the interferons and myelin basic protein are now being investigated. There is good reason to be optimistic about these approaches to therapy. If one can change the direction of the autoimmune process and regulate the effector cells away from the nervous system, one would hope that the disease process would then be halted.

Currently a number of immunosuppressive agents are being used. These include cyclophosphamide and azathioprine. These drugs are potentially toxic and the data to date is not clear that there is a beneficial therapeutic effect. The number of patients treated in controlled trials has been small but there are larger, more definitive trials underway.

Physical agents directed toward immune suppression have also been used. These include total body irradiation and plasma exchange. The strategy is the same as in the use of immunosuppressive agents, that is to produce immunosuppression that will hopefully reduce autoimmune reactivity directed toward the nervous system.

None of these agents have yet been proven to be effective in altering the long term natural history of MS. Most of them are under active investigation at this time and we expect that in the next few years objective evidence will be available concerning their possible effectiveness. Perhaps, the newer trials that are utilizing MRI as an adjunct measure of the extent of disease activity will produce convincing evidence either for or against the effectiveness of some of these agents.

\section{ACKNOWLEDGEMENTS}

Many thanks to my colleagues both at the University of British Columbia and elsewhere for helpful discussions and to Gerry Walker for her tireless help in typing the manuscript. The studies quoted in this paper were supported by the Medical Research Council, the British Columbia Health Care Research Foundation, the Canadian Multiple Sclerosis Society, and the Jacob W. Cohen Fund for research into Multiple Sclerosis.

\section{REFERENCES}

1. Ebers GC, Vinuela FV, Feasby T, Bass B. Multifocal CT enhancement in MS. Neurology 1984; 34: 341-346.

2. Raine CS, Scheinberg LC, Waltz JM. Multiple sclerosis-oligodendrocyte survival and proliferation in an active established lesion. Lab Invest 1981; 45: 534-536.

3. Prineas JW. Pathology of the early lesions of multiple sclerosis. Hum Pathol 1975; 6: 531-535.

4. Ludwin SK. Proliferation of mature oligodendrocytes after trauma to the central nervous system. Nature $1984 ; 308: 274-275$.

5. Prineas JW, Kwon EE, Sharer LR, Cho ES. Massive early remyelination in acute multiple sclerosis. Neurology 1987; 37 (Suppl 1): 109.

6. Paty DW, Isaac CD, Grochowski E, et al. Magnetic resonance imaging (MRI) in multiple sclerosis (MS): A serial study in relapsing and remitting patients with quantitative measurements of lesion size. Neurology 1986; 36 (Suppl 1): 177.

7. Willoughby E, Grochowski E, Li D, et al. A prospective study of MRI scanning in multiple sclerosis. Am Ac Neurol, New York 1987. Manuscript in preparation.

8. Palmer MR, Bergstron M, Grochowski E, et al. Magnetic resonance imaging (MRI) in multiple sclerosis (MS): Quantitative changes in the size of lesions over 6 months in the placebo limb of a therapeutic trial. Can J Neurol Sci 1986; 13: 168 .

9. Koopmans R, Li DKB, Grochowski E, et al. Benign and chronic progressive multiple sclerosis: An MRI study. Can J Neurol Sci $1987 ; 14: 241$.

10. Fog T, Linnemann F. The course of multiple sclerosis. Acta Neurol Scand 1970; 46 (Suppl 47): 1-175.

11. Stewart WA, Hall LD, Berry K, et al. Magnetic resonance imaging (MRI) in multiple sclerosis (MS): Pathological correlation studies in eight cases. Neurology 1986; 36 (Suppl 1): 320.

12. Noseworthy JH, Seland TP, Ebers GC. Therapeutic trials in multiple sclerosis. Can J Neurol Sci 1984; 11: 355-362. 